We.1.A.1.pdf

\title{
Fiber Raman amplification for metrological transfer of phase- coherent optical frequencies
}

\author{
G. Bolognini ${ }^{(1)}$, C. Clivati ${ }^{(2,3)}$, D. Calonico ${ }^{(2)}$, S. Faralli ${ }^{(4)}$, F. Levi ${ }^{(2)}$, A. Mura $^{(2)}$, N. Poli ${ }^{(5)}$ \\ (1) Consiglio Nazionale delle Ricerche, IMM Institute, 40129 Bologna, Italy, bolognini@bo.imm.cnr.it \\ (2) Istituto Nazionale di Ricerca Metrologica INRIM, 10135, Torino, Italy \\ (3) Politecnico di Torino, 10129, Torino, Italy \\ (4) Scuola Superiore Sant'Anna, TeCIP Institute, 56124 Pisa, Italy \\ ${ }^{(5)}$ Dip. Fisica e Astronomia, INFN and LENS, Università di Firenze, 50019 Sesto Fiorentino (FI), Italy
}

\begin{abstract}
We analyze the benefits of distributed Raman amplification in metrology transfer of phasecoherent ultra-narrowband optical frequencies in a long fiber link (200 km with additional $16 \mathrm{~dB}$ loss), providing high-gain bi-directional amplification with limited impact on phase noise, attaining a fractional frequency instability of $3 \times 10^{-} 19$ over $1000 \mathrm{~s}$.
\end{abstract}

\section{Introduction}

Metrological optical frequency standards are nowadays outperforming the primary cesium fountain clocks and are the most promising candidates for a redefinition of the second [1]. This improvement would be fruitless without a suitable method to compare such ultra-stable optical frequencies over long distances. Phase coherent optical fiber links can be used to this purpose [2-4], provided that phase noise introduced by the fiber is compensated together with optical losses. In such a compensation technique, light has to travel exactly the same path in both directions, with almost perfect bidirectionality, otherwise noise cancellation is not effective [4]. To overcome losses and preserve bi-directionality, special bi-directional Erbium Doped Fiber Amplifiers (BEDFA) [2,3,5] and Fiber Brillouin Amplifiers (FBA) [3,6] have been developed.

DRA has been employed for fiber-optic transmission $[7,8,10]$, but not yet in phasecoherent frequency metrology. This study involves an evaluation of gain and most common noise sources in DRA. We achieve DRA in a single-pump scheme on a metrological $200 \mathrm{~km}$ link, then in a double-pump scheme on $\sim 60 \mathrm{~dB}$ optical losses link, equivalent to $275 \mathrm{~km}$ fiber length, without intermediate amplification.

\section{Theory and DRA design}

The design of distributed Raman amplifiers in bidirectional configuration requires some care in order to exploit the DRA benefits without incurring in ongoing penalties, especially in extremely phase-sensitive metrological optical signals. In particular, the input optical power levels of the signal and Raman pumps need to be carefully optimized to avoid Kerr-related nonlinear effects (such as modulation instability or self-phase modulation), or the onset of stimulated Brillouin scattering. In addition, several noise source typical of DRA must be kept under control; among them polarizationdependent gain, double Rayleigh scattering (DRS), amplified spontaneous emission (ASE), and relative-intensity noise (RIN) transfer from pumps to signals [9]. A well-designed DRA should actually avoid such effects, as well as to maximizing the gain provided by the Raman amplification process, although in our phasecoherent transfer of ultra-narrowband signals, the above-mentioned effects are expected to bring a different impact with respect to standard wideband optical communication systems, and distortions in signal amplitude can induce phase distortions in transferred optical frequency through Kerr effect.

Our design is based on a fully resolved spectral model of Raman amplifiers describing the power evolution of co- and counter-propagating Raman pumps, an arbitrary number of signals, forward and backward ASE components and Rayleigh scattering effects [10], and optimization ensured that threshold power for nonlinear effects onset [7] is not reached along the fiber link. The design has first been carried out for signal carrier and counter-propagating pump, which is expected to provide most benefits in terms of gain and OSNR increase due to amplification of low-level signal, then considering both forward and backward directions simultaneously.

For the design, parameters for single-mode fibers of the actual link have been used (effective area $A_{\text {eff }}=80 \mu \mathrm{m}^{2}$, fiber loss equal to $\alpha_{\mathrm{s}}=0.22 \mathrm{~dB} / \mathrm{km}$ and $\alpha_{\mathrm{p}}=0.29 \mathrm{~dB} / \mathrm{km}$ at signal and pump wavelength respectively, a Raman gain coefficient $\left.g_{R}=3.8 \cdot 10^{-14} \mathrm{~m} / \mathrm{W}\right)$. Regarding counter-propagating DRA, figure 1a shows the calculated ON-OFF Raman gain (left y-scale), as well as OSNR related to ASE and DRS (left $y$-scale) for increasing values of pump power ($3 \mathrm{dBm}$ input signal power). The OSNR ASE (0.1 


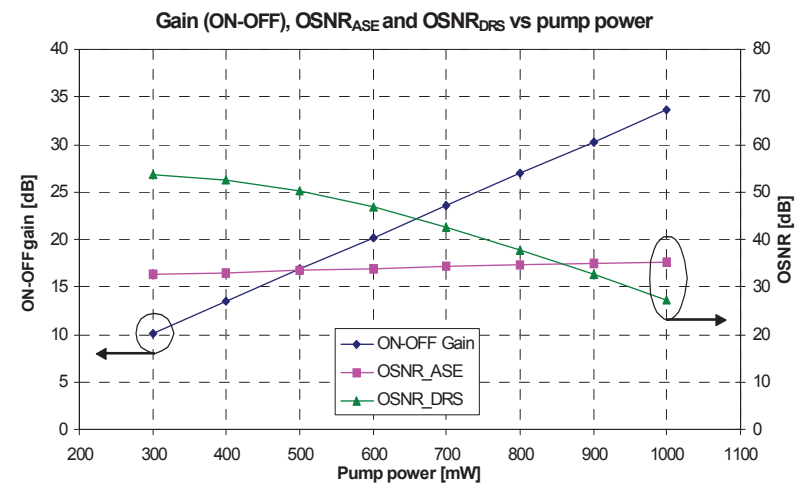

(a)

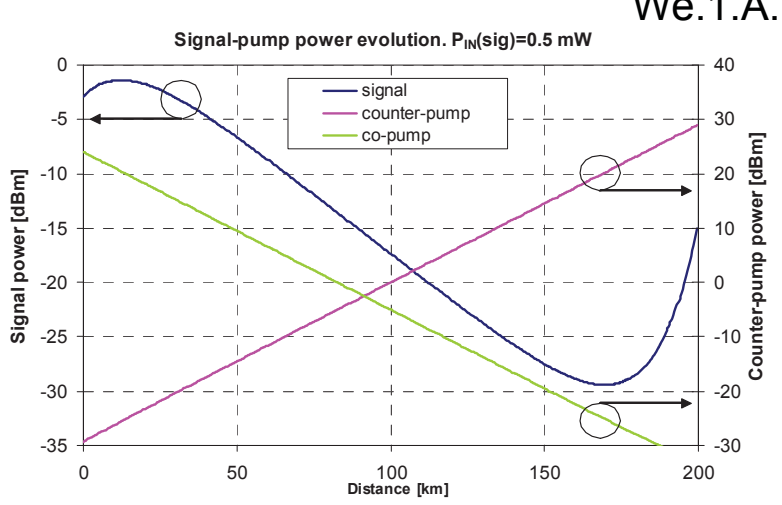

(b)

Fig. 1 (a) Simulated ON-OFF gain (left y-scale) and OSNR for ASE and DRS (right y-scale) versus counter-propagating pump power; (b) Calculated signal-pump power evolution inside the optical fiber for bi-directional pumping schemes.

$\mathrm{nm}$ bandwidth) and in-band OSNR DRS keep above $35 \mathrm{~dB}$ and $27 \mathrm{~dB}$ respectively, even with $34 \mathrm{~dB}$ gain.

The introduction of a co-propagating pump in addition to counter-propagating pump achieves bi-directional pumping, whose expected behaviour along the fiber can be inferred from Fig. $1 b$, reporting the power evolution for signal and pumps inside the optical fiber. The chosen optimal input signal power was $\mathrm{P}_{\mathrm{IN}}=0.5 \mathrm{~mW}$, in order to maximize the signal output at far fiberend, keeping at the same time the power level inside the fiber below the nonlinear threshold value (also allocating a 1-1.5 dB margin). The considered input power values for copropagating pump and counter-propagating pump are given by $\mathrm{P}_{\mathrm{IN}}(\mathrm{co}-\mathrm{pump})=260 \mathrm{~mW}$, (cnpump) $=500 \mathrm{~mW}$ respectively (due to maximum power available from our commercial lasers). Even when employing such high power levels, from the above-reported design no impairment (due to nonlinear effects, ASE or DRS) is expected to affect our optical frequency transfer experiment.

\section{Experimental set-up and results}

To study the DRA in a coherent optical link, we set up the laboratory test-bed shown in Figure 1. An ultrastable laser radiation at $1542 \mathrm{~nm}$ is generated by locking a fiber laser by PoundDrever-Hall technique to a Fabry-Perot cavity (finesse 120,000 ) made of Corning ULE ${ }^{\circledR}$ glass; the laser linewidth is $<30 \mathrm{~Hz}$ (details in [11]). The laser light is coupled into a standard single mode fiber (SMF-28), with a total length up to $200 \mathrm{~km}$, simulating a long-haul link from local to remote laboratory. The light power at fiber input is $0.5 \mathrm{~mW}$; total losses due to fiber and connectors amount to $\sim 45 \mathrm{~dB}$. To achieve phase noise cancellation, two acousto-optic modulators (AOM) are employed. AOM1, placed before the link, is used as actuator to compensate the fiber phase noise. AOM2, after the link, shifts the back-reflected signal, to distinguish between the signal (shifted by AOM2) and the backscattering (Rayleigh scattering is not shifted, whilst SBS is shifted by $\sim 10 \mathrm{GHz}$ ). Photodiode PD1 is employed to detect the phase error from an interferometric scheme [11]; then, a phase-locked-loop (PLL) feeds AOM1 with a correction signal. Photodiode PD2 is used to assess the link performance, comparing the phase noise of the signal at the remote end to that injected at the input by their beatnote. Residual pump radiation is filtered at detection by optical filters at 1542 $\mathrm{nm}(100 \mathrm{GHz}$ width) before PD1 and PD2

Co- and counter-pumped DRA is implemented by coupling the pump into the fiber through wavelength-division multiplexers WDM1 and WDM2 respectively. Two different pump lasers at $1450 \mathrm{~nm}$ are used. The first one is a depolarized fiber laser delivering up to $\sim 800 \mathrm{~mW}$ into the fiber. The second is composed of two polarization-multiplexed Fabry-Perot diode lasers, providing $\sim 260 \mathrm{~mW}$ [12]. Counterpumped DRA is less affected than co-pumped DRA by pump-signal RIN transfer, since it is

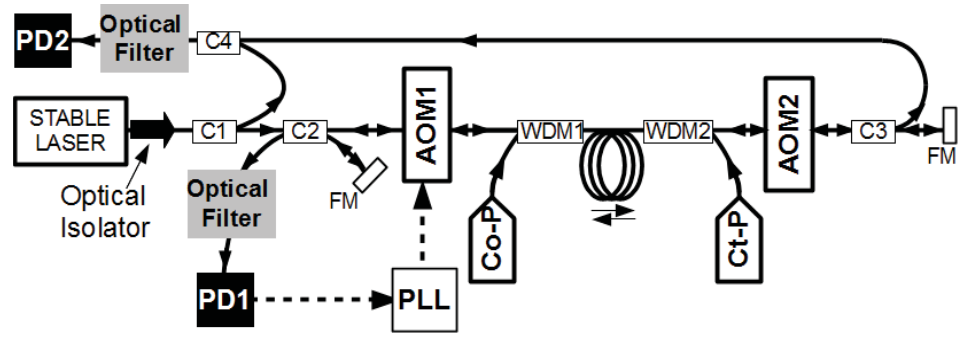

Fig. 2. Set up for DRA in a phase-coherent fiber link. PD Photodiode, C Couplers, FM Faraday Mirror, WDM Wavelength Division Multiplexer, Co-P Co-propagating Pump; Ct-P Counter-propagating Pump, AOM Acousto-Optic Modulator, PLL Phase Locked Loop. 


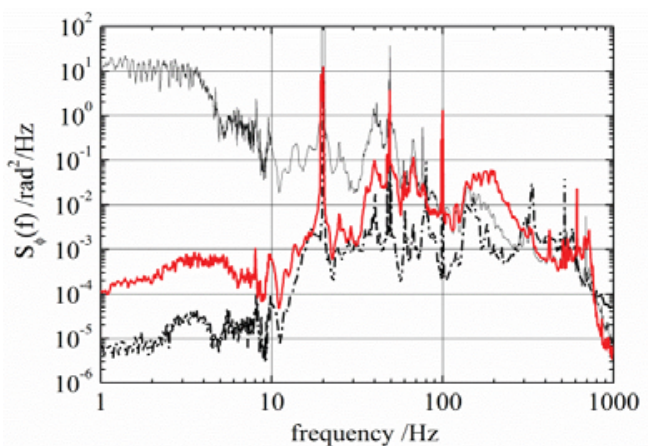

Fig. 3. Phase noise density $S_{\phi}(f)$ for the compensated link, at $100 \mathrm{~km}$ (dashed curve) and $200 \mathrm{~km}$ (solid), and non-compensated link at $200 \mathrm{~km}$ (upper curve).

averaged out by the large difference in pump and signal group velocities, [13]. We initially evaluate the DRA impact with a counter-pump scheme, using the fiber laser as pump. We detect on PD2 the heterodyne beatnote between the amplified signal and the light from the short reference arm. The absence of induced SBS noise, as expected from our design simulations, was confirmed by optical spectrum analysis of the transmitted signal and nearby frequency regions ( $25 \mathrm{GHz}$ span). In these conditions, we measure the phase noise density $S_{\phi}(f)$ and fractional frequency instability of the optical link (100 km and $200 \mathrm{~km}$ lengths). Figure $3 a$ shows the closed-loop phase noise at $100 \mathrm{~km}$ (dashed line), at $200 \mathrm{~km}$ (solid thick line), and also the phase noise for the uncompensated link at 200 $\mathrm{km}$ (solid thin line). The observed increase of the closed-loop noise from $100 \mathrm{~km}$ to $200 \mathrm{~km}$ at frequencies much smaller than the locking bandwidth is in agreement with the theoretical predictions taking into account the fiber length increase, i.e. $S_{\phi}(f) \propto L^{3}[4]$. Link stability over long periods is calculated by counting the beatnote on PD2 with a $\Lambda$-type counter [14]. The data instability $\sigma_{\Lambda}(T)$ obtained with a $\Lambda$-type counter can be converted into the Modified Allan deviation $\bmod \sigma_{A}(T)$ for common phase noises [15]. Figure $3 \mathrm{~b}$ shows $\sigma_{\Lambda}(T)$ of the link at $100 \mathrm{~km}$ (circles), at $200 \mathrm{~km}$ (squares) and of the interferometer without fiber spools (diamonds). Up to $20 \mathrm{~s}$ white phase noise is dominant, thus $\bmod \sigma_{A}(T) \sim 0,35 \times \sigma_{A}(T)$, , whilst after $20 \mathrm{~s}$

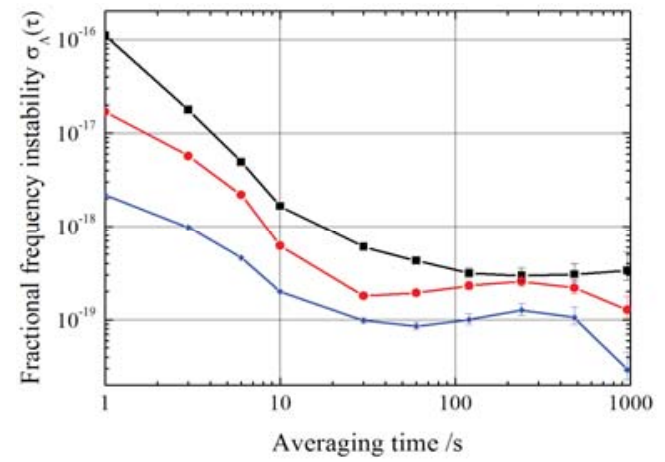

Fig. 4. Fiber link frequency instability using bidirectional DRA: $100 \mathrm{~km}$ (circles), $200 \mathrm{~km}$ (squares), interferometer noise floor (diamonds) frequency flicker noise is dominant and $\bmod \sigma_{A}(T) \sim 0,7 \times \sigma_{\Lambda}(T)$. At $200 \mathrm{~km}$ with counterpumped DRA, the instability is $\sigma_{\Lambda}(T) \sim 3 \times 10^{-19}$ from $100 \mathrm{~s}$ to $1000 \mathrm{~s}$, limited by the fiber residual non-reciprocal noise. Counter-pumped DRA appears fully reliable for a phase coherent optical link. To further increase the fiber span, a bi-directional pump scheme is tested, adding the Fabry-Perot diode laser, coupled into the fiber through WDM1, as a co-pump. DRA overall gain was then increased to $32 \mathrm{~dB}$, equivalent to a single stage DRA with $1 \mathrm{~W}$ single counter-pump [10], limited by the available diode laser power $(260 \mathrm{~mW})$. We observed that the double stage pump allows to implement the link with $61 \mathrm{~dB}$ losses, equivalent to a $275 \mathrm{~km}$ fiber haul, obtained inserting a $16 \mathrm{~dB}$ attenuator.

In conclusion, we showed that DRA is suitable for phase coherent optical links, allowing the optical frequency transfer on $275 \mathrm{~km}$ in single fiber span, with a frequency instability of $3 \times 10^{-19}$ at $100-1000 \mathrm{~s}$. ROA offers several advantages with respect to other amplification techniques; however, particular care should be devoted to the design of links involving both counter- and co-pumping, to avoid nonlinear scattering affecting the ultimate performance.

\section{References}

1. C. W. Chou et al., Phys. Rev. Lett. 104, 070802 (2010).

2. O. Lopez et al., Opt. Expr. 18, 16849-57 (2010).

3. K. Predehl et al., Science 336, 441-444 (2012).

4. W. Williams et al., J. Opt. Soc. Am. B 25, 1284-1293 (2008).

5. E. Desurvire, Erbium-doped fiber amplifiers: principle and applications, Wiley-Interscience publisher (1994).

6. O. Terra et al., Opt. Expr. 18, 16102-10 (2010).

7. G. Agrawal, Nonlinear Fiber Optics (Academic Press, 2001).

8. M. Islam, IEEE J. Sel. Top. Quantum Elect. 8, 548-559 (2002).

9. R. H. Stolen, in Tech. Dig. Symp. Optical Fiber Measurements, NIST Special Publication 953, 139 (2000)

10. S. Faralli, et al., IEEE J. Lightwave Technol. 23, 2427 (2005)

11. C. Clivati, et al., IEEE Trans. Ultrason. Ferroelectr. Freq. Contr. 58, 2582-2587 (2011).

12. G. Bolognini et al., IEEE Photon. Technol. Lett. 18, 1591 (2006).

13. C. R. S. Fludger et al., J. Lightwave Technol., 19, 1140-1148 (2001)

14. E. Rubiola, Rev. Sci. Instrum. 76, 054703 (2005).

15. S. T. Dawkins et al., IEEE Trans. Ultrason. Ferroelectr. Freq. Control 54, 918-925 (2007). 